\title{
Reconstructing Galaxy Spectral Energy Distributions from Broadband Photometry
}

\author{
I. Csabai ${ }^{1}$, A. J. Connolly ${ }^{2}$, A. S. Szalay ${ }^{1}$ \\ Department of Physics and Astronomy, The Johns Hopkins University, Baltimore, MD 21218 \\ Electronic mail: csabai@skysrv.pha.jhu.edu, ajc@skysrv.pha.jhu.edu, szalay@skysrv.pha.jhu.edu \\ and \\ T. Budavári ${ }^{3}$ \\ Department of Physics, Eötvös University, Budapest, Pf. 32, Hungary, H-1518
}

\begin{abstract}
We present a novel approach to photometric redshifts, one that merges the advantages of both the template fitting and empirical fitting algorithms, without any of their disadvantages. This technique derives a set of templates, describing the spectral energy distributions of galaxies, from a catalog with both multicolor photometry and spectroscopic redshifts. The algorithm is essentially using the shapes of the templates as the fitting parameters. From simulated multicolor data we show that for a small training set of galaxies we can reconstruct robustly the underlying spectral energy distributions even in the presence of substantial errors in the photometric observations. We apply these techniques to the multicolor and spectroscopic observations of the Hubble Deep Field building a set of template spectra that reproduced the observed galaxy colors to better than $10 \%$. Finally we demonstrate that these improved spectral energy distributions lead to a photometric-redshift relation for the Hubble Deep Field that is more accurate than standard template-based approaches.
\end{abstract}

Subject headings: galaxies: photometry — galaxies: distances and redshifts

\section{Introduction}

With the application of photometric-redshifts to wide-angle, multicolor photometric surveys the study of galaxy evolution has moved from expressing the evolution as a function of observable

\footnotetext{
${ }^{1}$ Department of Physics, Eötvös University, Budapest, Pf. 32, Hungary, H-1518

${ }^{2}$ Department of Physics and Astronomy, University of Pittsburgh, Pittsburgh, PA 15260

${ }^{3}$ Department of Physics and Astronomy, The Johns Hopkins University, Baltimore, MD 21218
} 
parameters (e.g. magnitudes and colors) to one where we can describe the evolution of galaxies in terms of their physical attributes (i.e., their redshift, luminosity and spectral type). Over the last several years with new multicolor surveys coming on-line these techniques have become increasingly popular enabling large, well defined statistical approaches to galaxy evolution.

In the astronomical literature there are a number of different approaches to estimating the redshifts of galaxies from their broadband photometry. While these techniques differ in their algorithmic details they share the same underlying goal. We wish to model the change in galaxy color as a function of redshift (and galaxy type) and use these models to estimate galaxy redshifts, in a statistical sense. For simplicity we divide the differing techniques into two classes, those which use spectral energy distributions (whether derived from models or empirically from observations of local galaxies) as spectral templates and those which derive a direct empirical correlation between color and redshift using a training set of galaxies.

Template based photometric-redshifts are constructed by comparing the observed colors of galaxies to a set of galaxy spectral energy distributions. Their strength is that they are simple to implement and can be applied over a wide range in redshift. Their main limitation is that we must know the underlying spectral energy distributions of galaxies within our sample. Comparisons of the colors of galaxies with spectral synthesis models (Bruzual and Charlot 1993) have shown that the modeling of the ultraviolet part of galaxy spectra is highly uncertain (whether this is due to uncertainties in the modeling of the stars or due to the effect of dust is unclear). Consequently, photometric-redshift estimates are most accurate when we apply empirical spectral energy distributions derived from observations of local galaxies (e.g. Sawicki et al 1997). These empirical relations are however constructed from only a handful of galaxies that have been observed in detail and there is no guarantee that they represent the full distribution of galaxy types (particularly when we include the effects of evolution with redshift).

The second approach is to derive a direct correlation between the observed colors of galaxies and their redshifts using a training set that contains spectroscopic and photometric data. The strength of this technique is that the relation is purely empirical; the data themselves define the correlation between color and redshift. The effects of dust and galaxy evolution that are present in the training set are, therefore, implicit within the derived correlation. Their weakness is that the correlations cannot be extrapolated to redshifts beyond the limits of the training set and that a sample of galaxies with redshifts (and selected to have a broad color distribution) must be present before the photometric-redshift relation can be derived.

Clearly if we can combine these two approaches we may derive the optimal approach for estimating galaxy redshifts. If we can use a training set of galaxies to define the underlying spectral energy distributions (which will include the effects of evolution and dust) then we can apply these empirical template spectra over a wide range in redshift. In this paper we describe a fundamentally new approach to photometric-redshifts that extends our previous work on estimating galaxy redshifts from broadband colors such that we construct spectral energy distributions directly 
from the broadband data. In Section 2 we outline the physical and mathematical basis of this new approach. In Section 3 we apply these techniques to a sample of galaxies with simulated colors, showing that we can recover the underlying spectral energy distributions that describe these galaxies. Section 4 applies the optimization procedure to the multicolor photometric data of the Hubble Deep Field (Williams et al 1996) and shows that this technique can be used to improve the accuracy of template-based photometric redshift relations. Finally in Section 6 we describe the application of these techniques to analysis and the modeling of galaxy spectral energy distributions.

\section{Building Spectral Templates from Broadband Photometry}

In an earlier work (Connolly et al 1995a) we described how to model the empirical correlation between the colors of galaxies and their redshifts by fitting a multi-dimensional polynomial relation. This technique proved successful for estimating the redshifts of galaxies in the $0<z<1$ regime but was built on a very general but somewhat unphysical basis that the color-redshift relation can be described by a low order polynomial. Ideally we want the underlying basis on which we define the photometric-redshift relation to be physically motivated. If we can construct a set of low resolution spectral energy distributions directly from a set of galaxies with multicolor photometry then we can achieve this goal (an empirical, physical basis).

If we consider a galaxy, at a redshift $z$, observed through a series of broadband filters then the restframe fluxes observed through each filter, $f_{k}$ can be described by,

$$
f_{k}=\int R_{k}(\lambda) S(\lambda /[1+z]) d \lambda
$$

where $R_{k}(\lambda)$ is the response function of the $k$ th filter and $S(\lambda /[1+z])$ is the spectral energy distribution of a galaxy blueshifted to the galaxy's restframe. The response function, $R_{k}(\lambda)$, includes not only the filter transmission but also the instrumental and observational effects such as the CCD quantum efficiency and the change in effective shape of the filter due to absorption by the atmosphere. The spectral energy distribution, $S(\lambda /[1+z])$, is the true underlying spectrum that includes the stellar composition of the galaxy together with the effect of intragalactic extinction. For high redshift objects the effects of the IGM should be built in the above equation.

We can see that $f_{k}$ is nothing more than a convolution of the input spectrum with the filter response function. Thus from a photometric catalog of galaxies with identical restframe spectral energy distributions, given the filter response functions, we can deconvolve the underlying spectral energy distribution. More exactly we can recover a low resolution slice of the spectrum whose limits are defined by the wavelength range over which the filters extend and the redshifts of the galaxies in the catalog.

If we observe just one object there are, of course, a wide range of spectra that could give the exact flux values passing through the broadband filters. The question then arises, if instead we 
have an ensemble of $N$ galaxies over a range of redshift, with accurate redshifts and $K$ multicolor photometric observations per galaxy, can we invert this relation to recover the underlying spectral energy distributions (even in a statistical sense)? In principle, for a sample of galaxies spread over a range in redshift, we have $N \times K$ measurements of the underlying spectral energy distributions. For a series of optical and near-infrared filters the resolution of our reconstructed spectra would be proportional to the rest wavelength range sampled by the filters divided by $N \times K$.

The advantage of this technique is that it is numerically straightforward to calculate. The deconvolution algorithms do not require a large amount of computational power. Its main weakness is that in the real world we cannot construct a large catalog of galaxies with identical spectral energy distributions on which to apply the deconvolution algorithm. We can circumvent this, however, by noting that galaxy spectra can be described by a small number of orthogonal spectral components or eigenspectra (Connolly et al 1995b). Each galaxy spectrum can be written as,

$$
S(\lambda)=\sum_{j=1}^{J} a_{j} E_{j}(\lambda)
$$

where $E_{j}(\lambda)$ are the $J$ eigenspectra and $a_{j}$ are the expansion coefficients. Each galaxy spectral type can then be described by a linear combination of the eigenspectra (i.e. only the expansion coefficients, $a_{j}$, differ as a function of spectral type). From observations of local star forming and quiescent galaxies it has been shown that the number of components (or eigenspectra) required to reconstruct the continuum shape of a galaxy spectrum (to an accuracy of better than 1\%) is small, typically $2-4$ (Connolly et al 1995b).

Utilizing the eigenspectra formalism simplifies the deconvolution problem in two ways. By restricting the number of components we need to reconstruct from the multicolor data to 2-4 the inversion process is much simplified. Secondly, the fact that any galaxy spectrum can be described by this small number of components enables us to use all of the available data (i.e. we do not have to restrict our analysis to a single class of galaxies with similar spectral types).

\subsection{Template spectrum estimation}

The goal of the deconvolution is to provide a set of $J$ eigenspectra, $E_{j}(\lambda)$, that best represent the observed colors of the galaxy training set. We can parameterize the eigenspectra as a linear combination of basis functions such that,

$$
E_{j}(\lambda)=\sum_{l=1}^{L} b_{j l} B_{l}(\lambda),
$$

where $B_{l}(\lambda)$ are a set of $L$ basis function vectors (e.g. Legendre polynomials) and $b_{j l}$ are their relative expansion coefficients. In this paper we choose to parameterize the eigenspectra in terms of a linear combination of Legendre polynomials. The choice of the parameterization is, however, 
completely arbitrary. There are many ways we could describe the galaxy eigenspectra. In the simplest case the coefficients $b_{j l}$ could be just the flux values of the eigenspectra measured at a fixed set of wavelengths (i.e. the the basis functions would be delta functions centered at these wavelengths). We would then be reconstruct the eigenspectra directly rather than expressing it in terms of a linear combination of functions (Budavari et al 1999). We also note that, while we use the term eigenspectra, the above procedure does not guarantee (nor is it necessary) that the eigenspectra be orthogonal.

From Equations 1, 2 and 3 we can now estimate that color of a galaxy in terms of the linear combination of eigenspectra or basis functions. For the $i$ th galaxy in a ensemble of photometric observations the estimated flux through the $k$ th filter is given by,

$$
\begin{aligned}
f_{i k}^{e} & =\int_{0}^{\infty} R_{k}(\lambda) \sum_{j=1}^{J} a_{i j} E_{j}\left(\lambda /\left[1+z_{i}\right]\right) d \lambda \\
& =\int_{0}^{\infty} R_{k}(\lambda) \sum_{j=1}^{J} \sum_{l=1}^{L} a_{i j} b_{j l} B_{l}\left(\lambda /\left[1+z_{i}\right]\right) d \lambda
\end{aligned}
$$

We can now define a $\chi^{2}$ or cost function that describes the distance between the observed flux values, $f_{i k}^{m}$, measured for particular galaxy and those predicted by the eigenspectra, $f_{i k}^{e}$. We write the form of the cost function as a $\chi^{2}$, weighted by the measured flux errors $\sigma_{i k}$ but other distances can be also used, dependent on how one would like to weight the different observations.

$$
\chi^{2}=\sum_{i=1}^{N} \sum_{k=1}^{K} \frac{\left[f_{i k}^{e}-f_{i k}^{m}\right]^{2}}{\sigma_{i k}^{2}} .
$$

The cost function depends on parameters $a_{i j}$ and $b_{j l}$. The minimum of this cost function determines the set of optimal parameters (in other words eigenspectra and expansion coefficients) that give the best estimation of fluxes in this framework. Of course, the larger the catalog of galaxies with multicolor observations the more non-redundant parameters we can optimize for and, consequently, the finer the resolution in the eigenspectra.

By carefully choosing how we generate the eigenspectra we can have a cost function whose minimum can be found almost analytically. The variable parameters of the cost function will be $a_{i j}$ and $b_{j l}$. Since Equation 5 is linear in both of them they will appear in a quadratic form in the $\chi^{2}$ cost function. At the $\chi^{2}$ minimum all of the derivatives of the cost function should be zero. If we consider the values $a_{i j}$ as constants, the equations with the derivatives in $b_{j l}$ will give a set of $J \times L$ linear equations. In similar way keeping $b_{j l}$ constant we will have $N \times J$ linear equations for $a_{i j}$, or to be more exact it breaks up into $J$ sets of $N$ linear equations with $N$ unknowns in each. Each of these set of linear equations can be solved independently. Therefore, by iteratively solving the two sets of linear equations (holding one set of coefficients constant while solving for the other coefficients) one can minimize the cost function in a efficient manner. 
In fact one could generate the eigenspectra in many other ways, for example, using a tanh() function to mimic the $4000 \AA$ break and a power law function to represent the star formation at the ultraviolet end of the spectrum. Parameters could occur in the cost function in more complex forms than showed above. If the variable parameters are not in a quadratic form in the cost function nonlinear optimization methods (such as different types of gradient descent methods or simulated annealing if local minima cause problems) could be used instead of solving linear equations. This would be computationally harder but the shape of the eigenfunctions would not be restricted to a particular basis.

The limitation on the number of parameters and, therefore, the number of Legendre polynomials, is defined by the set of galaxies with multicolor photometric observations. As described previously, if we have $N$ galaxies with observations in $K$ passbands then we have $N \times K$ independent measurements. If we want to describe the distribution of galaxy types by $J$ eigenspectra (in our case 3 eigenspectra) then we have $J \times N$ constraints. This means that the number of degrees of freedom in the system and, therefore, the maximum number of parameters (or polynomials) we can solve for, $X$, is,

$$
X \leq \frac{N(K-J)}{J}
$$

As we noted earlier the parameterization we choose to describe the eigenspectra by is completely arbitrary. A second, and more physical, way to visualize how the number of observations (number of galaxies and passbands ) relates to how well we can recover the underlying eigenspectra is to think of the parameters $X$ as the number of wavelengths at which we can sample a galaxy spectrum. This involves using the values $E_{j}\left(\lambda_{l}\right)$ of a discretely sampled low resolution eigenspectrum as optimization parameters. The basis functions will, therefore, have a constant value 0 except at a selected $\lambda_{l}$ where the value is 1 . In such a way we have a direct, low resolution realization of the underlying eigenspectra that describe the observed galaxy population. It is then clear that, given the wavelength interval we wish to reconstruct, $X$ defines the maximum resolution of the resultant eigenspectra.

The number of observations and the passbands are given by the measurements but we have some freedom to chose the number of eigenspectra and the resolution. For example if we want to use the templates for photometric redshift estimation we have to have enough resolution to reconstruct the broadband features of the spectrum. So we need resolution at least the width of the (blueshifted) filters. On the other hand to represent all different spectral types even rare ones we would like to have large number of templates. If the number of observations is not enough to satisfy both of these requirements one has to chose between representing equally the spectra of all of the objects but with poor quality or to represent the typical ones with better resolution and have a few outliers. Comparing the final value of the cost function or the quality of photometric redshift estimation can help to set these parameters optimally. 


\section{Application to Simulated Data}

To demonstrate the validity of this technique and to determine the accuracy to which we can reconstruct galaxy spectral energy distributions from broadband photometry we initially apply the algorithm to a set of simulated data. From the Bruzual and Charlot spectral synthesis models (BC96, Bruzual and Charlot 1995) we construct a set of galaxy spectra using a simple stellar population ranging in age from 0 yrs to 20 Gyrs (with solar metalicity and a single burst of star formation). In total the sample contains 222 spectra covering the spectral range $200 \AA$ to $2.2 \mu \mathrm{m}$. From these spectra we apply a Principal Component Analysis or Karhunen-Loève transform (Karhunen 1947, Loève 1948, Connolly et al 1995b) to construct a series of orthogonal eigenspectra. The first two of these eigenspectra are shown in Figure 1a and Figure 1b respectively.

Using the first two eigenspectra we simulate the colors of galaxies as a function of redshift. The expansion coefficients $a_{i}$, or mixing angles, are designed to produce a set of galaxy colors that match the color distribution of galaxies within the local Universe. In total, we construct a sample of 616 galaxies with $\mathrm{U}, \mathrm{B}, \mathrm{V}, \mathrm{I}, \mathrm{J}, \mathrm{H}$ and $\mathrm{K}$ photometry covering the redshift range $0<z<1.35$. The upper limit on the redshift range is imposed for two reasons, to match the redshift distribution of those galaxies in the Hubble Deep Field with $V_{606}<24$, and secondly to avoid the added complication of including the attenuation due to the intergalactic medium (e.g. Madau et al 1996).

\subsubsection{Reconstructing Bruzual and Charlot Spectra}

Given these simulated data (colors and redshift) we use the algorithms described in Section 2 to reconstruct the underlying eigenspectra. The redshift distribution of the input catalog of

galaxies defines the wavelength range over which the eigenspectra can be reconstructed (the upper and lower bounds being defined by the restframe upper and lower filter cutoffs of the $\mathrm{K}$ and $\mathrm{U}$ passbands respectively). For the redshift range of the Bruzual and Charlot data we can recover the spectral energy distribution over a wavelength interval of approximately $950<\lambda<22000 \AA$.

Initially we sample the eigenspectra that we wish to recover in 20 bins (sampled linearly in wavelength), with a spectral resolution of approximately $1000 \AA$. Each of the two approaches (i.e. using Legendre polynomials and evenly sampled random spectra) outlined in Section 2 were applied to the data and were found to give identical results. In the section below we will, therefore, only discuss the optimization of the eigenspectra using Legendre polynomials. A subsequent paper (Budavari et al. 1999) will discuss in more detail techniques that can be applied directly to the spectra themselves.

For basis functions, $B_{l}(\lambda)$, we used the first 20 Legendre polynomials. The optimization procedure was started with the Legendre polynomials, $B_{l}(\lambda)$, specified by a series of random numbers. The optimization was found to converge rapidly for the 616 galaxies within our simulated 
data. After 50 iterations, a few minutes of workstation cpu time, the cost function was found to be stable (varying by less than $0.1 \%$ from one iteration to the next). The rapid convergence of the relative error as a function of iteration is shown in Figure 2.

Comparison between the eigenspectra input into the simulations and the spectra reconstructed by our optimization technique is not straightforward. While the spectral templates that the reconstruction technique derives should occupy the same subspace as the original eigenspectra there are many non-unique ways to achieve this (i.e. the output spectra can be a rotated subset of the input spectra). To transform the input and output spectral templates into a common form, for each input eigentemplate, we calculate the linear combination of the output eigentemplates that gives the closest representation of input eigenspectrum (i.e. we project the original eigenspectra onto the reconstructed eigenbasis). This produces a set of eigenspectra that can then be directly compared with the BC96 input basis. In Figure 1 we show a comparison between the BC96 eigenspectra and those we derive from the optimization technique. Clearly there is an almost perfect one-to-one match between the two with the rms scatter less than $7 \%$ both for the first and second eigenspectra.

The analysis we show in Figure 1 is the ideal case (where the noise in the observations is negligible). As deep photometric and spectroscopic surveys tend to push analyses of the data to the limit of the survey we need to investigate the effect of photometric uncertainties on the reconstruction of the underlying spectral energy distributions. For simplicity we assume a constant photometric error across all passbands (i.e. we do not allow for the lower signal-to-noise that are prevalent in ultraviolet observations of intermediate redshift galaxies).

Figure 3 shows the effect of increasing the photometric uncertainty on the reconstruction of the first eigenspectrum. The solid line is the reconstructed eigenspectrum with no noise added, the triangles, squares, and circles show the effect of adding 5\%,10\% and $20 \%$ flux errors respectively. As we see even with very low signal-to-noise data the eigenspectra can be reproduced to a very high accuracy. The large number of galaxies present within the sample means that each spectral interval (i.e. the $1000 \AA$ spectral bins) is sampled by multiple galaxies. The coaddition of these multiple realizations increases the signal-to-noise of the reconstructed spectrum (relative to the input data).

We note that for a flux error in excess of $5 \%$ the long wavelength end of the eigenspectrum becomes significantly more noisy than the remaining spectral regions. This arises because the longest rest wavelengths are only sampled by the lowest redshift galaxies. Therefore, the reconstructed spectrum will have a larger uncertainty where the spectral values are constrained by only a small number of data points. For decreasing signal-to-noise the longest wavelength spectral regions will be the most susceptible to the effect of the noise and can, therefore, be used as an indicator of when photometric uncertainties become significant within an analysis. 


\subsubsection{Photometric redshifts from empirical eigentemplates}

Having reconstructed the eigenspectra that describe the distribution of galaxy colors we utilize these spectra to derive a photometric redshift relation. We note that the reconstruction technique does not involve minimizing the difference between the spectroscopic redshift of a galaxy and its photometric redshift rather it minimizes the differences in the observed and estimated colors. This means that the dispersion about the photometric redshift relation is an accurate measure of how well the reconstructed spectra match the simulated data.

We apply the standard template-based photometric redshift relation, adapted to utilize eigenspectra (e.g. Benitez 1999). For the range of redshifts we wish to consider (in the case of our simulations $0<z<1.35)$ we define a redshift dependent $\chi^{2}(z)$,

$$
\chi^{2}(z)=\sum_{i=1}^{N} \sum_{k=1}^{K} \frac{\left(f_{i k}^{m}-\sum_{j} a_{j} E_{j k}(z)\right)^{2}}{\sigma_{i k}^{2}}
$$

where $f_{i k}^{m}$ is the color of galaxy $i$ observed through the $k$ th filter, $\sigma_{i k}$ is the flux error, $a_{j}$ are the expansion coefficients of the eigensystem (which we also solve for) and $E_{j k}$ is the color of the $j$ th eigenspectrum observed through the $k$ th filter. Minimizing this relation gives the estimated redshift of the galaxy (and is a simple and fast 1 dimensional problem).

While the deconvolved eigentemplates are found to be marginally susceptible to the effects of photometric uncertainties within the data we find that the dispersion in the resulting photometric redshift relation derived from these spectral templates is sensitive to the photometric noise. In Figure 4 we show the dispersion in the photometric redshift relation for a set of simulated data with photometric uncertainties of $0 \%, 5 \%, 10 \%$ and $20 \%$. For each sample the eigenspectra were derived directly from the data themselves (i.e. including the photometric errors). These eigenspectra were then used to derive the photometric-redshift relation. The top left panel shows the photometric-redshift relation in the presence of no noise $\left(\sigma_{z}=0.018\right)$, the top right the effect of $5 \%$ flux error $\left(\sigma_{z}=0.049\right)$, the bottom left the effect of $10 \%$ flux errors $\left(\sigma_{z}=0.104\right)$, and the bottom right the effect of $20 \%$ flux errors $\left(\sigma_{z}=0.258\right)$.

The correlation between the dispersion about the photometric-redshift relation and the signal-to-noise of the data is not, however, due to errors present within the reconstructed

eigenspectra. We demonstrate this in Figure 5. We derive the eigenspectra for a sample of galaxies with $20 \%$ flux errors (i.e. relatively low signal-to-noise). We then use these eigenspectra in a photometric-redshift analysis of a set of data with no flux errors. The resultant relation has a dispersion of 0.051 (substantially smaller than the 0.25 derived from the simulations with $20 \%$ flux errors). The limitation on the accuracy of a photometric redshift is, therefore, almost entirely due to the signal-to-noise of the data set to which we wish to apply the relation.

\section{Deriving Spectral Energy Distributions from Broadband Photometry}




\subsubsection{Templates from Multicolor Photometric Observations}

The most natural data set on which to apply our deconvolution techniques are the Hubble Deep Field observations (HDF; Williams et al 1996). These data comprise a single WFPC2 field observed in four ultraviolet/optical passbands (F300W, F450W, F606W and F814W). The HDF has been the target of a substantive effort to obtain deep spectroscopic redshifts and follow up near-infrared and longer wavelength imaging for the central and flanking fields. It represents one of the densest regions of the sky in terms of published multicolor photometry and spectroscopy. In total there are over 110 galaxies within the HDF with high signal-to-noise optical and near-infrared colors and redshifts.

\subsection{Template estimation}

To enable a direct comparison between the accuracy of photometric-redshifts based on our template estimation algorithm and those derived by others from standard techniques we use the photometric catalog of Fernandez-Soto et al (1999). These data are based on the optical WFPC2 colors and followup ground-based near-infrared imaging ( $\mathrm{J}, \mathrm{H}$ and $\mathrm{K}$ ) of the HDF (Dickinson et al 1999). From this catalog we extract the 74 galaxies with spectroscopic redshifts, $z<1.35$, and with broadband optical and near infrared magnitudes (a total of 7 passbands). The upper redshift limit is imposed on our galaxy selection to remove the effect of the IGM on the observed colors of the galaxies (which comes into effect at $z>2$ ).

An other reason to limit most of our study to this redshift range is that the number of galaxies with known redshift and reliable photometry is small. Using higher redshifts would force us to extend the wavelength range of the reconstructed templates on the low wavelength end. This part of the templates would be estimated based on the information from a small number of high

redshift galaxies (usually with larger photometric errors) resulting larger errors in the shape of the templates.

For the deconvolution we assume the filter curves and CCD quantum efficiency curves that are publicly available at the STScI and National Optical Astronomical Observatories websites. We note in passing that the resolution of the quantum efficiency curve of the infrared camera (IRIM) used for the near infrared observations is poorly known (i.e. the resolution is poor) and that a better representation of this curve may improve on the accuracy of the results we present below.

Using these data we apply the optimization algorithm described in Section 2. The redshift range of the data coupled with the filter set enables us to reconstruct the spectral energy distributions over a wavelength range of 976-21946 A. Within this interval we reconstruct 3 eigenspectra described by 20 Legendre polynomials (sampled at a resolution of $450 \AA$ ). The resultant spectral energy distributions, after approximately 50 iterations, are shown in Figure 6. The solid line shows the first eigenspectrum and the dashed and dotted lines the second and 
third eigenspectra respectively. The relative flux error (the deviation between the colors we would estimate based on the three eigenspectra and those measured for each galaxy) is less than $9 \%$. It is quite remarkable that with only 3 eigenspectra and a poor spectral resolution we could reconstruct the fluxes with errors that are comparable with the errors in the observations.

The limit on the resolution of the reconstruction is the number of galaxies with redshifts and multicolor photometry. For the 74 galaxies within the HDF sample, observed in 7 passbands, there are a total of 296 degrees of freedom (for 3 eigenspectra - see Equation 7). The 20 Legendre polynomials used in each of the 3 eigenspectra add up to a total of 60 parameters that we must solve for. The current solution is, therefore, well constrained. With a larger sample of galaxies (as we describe in Section 2.1) we could improve on the spectral resolution.

Even given the poor resolution of the reconstructed spectra $(\sim 450 \AA)$ there are a number of noticeable spectral features present within the reconstructed data. The first eigencomponent (essentially the mean of the galaxy distribution) has the spectral shape of a Sbc or Scd galaxy (cf. the Coleman Wu and Weedman galaxy spectral energy distributions). At the redshifts we probe $(\bar{z}=0.8)$ this is consistent with the median galaxy type (Lilly et al 1995). In the first eigenspectrum there is also clear evidence for a break in the galaxy spectrum at $4000 \AA$ (due to Balmer series absorption). The second and third eigenspectra appear to be dominated by emission in the ultraviolet part of the spectrum with a strong upturn at $\lambda<4000 \AA$, consistent with a star forming population. In fact the distribution of the spectral continuum shape of the eigenspectra are consistent with those we derive from both the BC96 models and from observations of local galaxies.

For wavelengths greater than $1.4 \mu \mathrm{m}$ the reconstructed spectra suffer from a ringing in the reconstruction. As we noted previously, because the information used in reconstructing this spectral region is derived only from the lowest redshift galaxies within our sample then the long wavelength regions will have lower signal-to-noise. Consequently these long wavelength regions are more susceptible to the limited number of galaxies used in the reconstruction (i.e. the first signs of the error show up here). We note, however, that when calculating magnitudes from these spectral templates the convolution with the filters partially averages out these fluctuations.

\section{Photometric-Redshifts from Empirical Spectral Energy Distributions}

While a comparison between how well we can reconstruct the observed colors of galaxies using the empirical spectral templates is a measure of the "goodness-of-fit" the goal of this technique is to improve the accuracy of the photometric redshift relation. We, therefore, compare the photometric redshift-relation we derive using these empirical eigentemplates with those relations given in the literature (Sawicki et al 1996, Gwyn and Hartwick 1996, Fernandez-Soto et al 1999).

Principally we concentrate on the redshift estimators of Fernandez-Soto et al (1999), for which we have an identical set of photometric observations. 
Using the eigentemplates as spectral energy distributions we use the standard template fitting method to derive a sample of photometric redshifts. A comparison between the estimated and spectroscopic redshifts, for the redshift range $0<z<1.35$, is given in Figure 7 . The solid line represents a one-to-one comparison between the photometric and spectroscopic redshifts. About this line the rms dispersion within the relation is $\sigma_{z}=0.077$ using three eigencomponents. This compares favorably with the results of Fernndez-Soto et al. (1999) who achieve a dispersion of $\sigma_{z}=0.095$ using the four Coleman, Wu and Weedman (1980) (CWW) spectral energy distributions and also with the polynomial fitting techniques of Connolly et al. (1995) who determine an $\sigma_{z}=0.14$ and $\sigma_{z}=0.062$ with first and second order polynomial fits respectively.

Although the number of galaxies with $z>1.5$ is small to get reliable templates for the high redshift galaxies we made a test to demonstrate that the method does not brake over this redshift limit (Figure 8.). The higher redshift range increase the number of observations to 102 from the original 74 but the wavelength range needed for the templates also extends. Since higher redshifts strech the restframe spectra more, to get the same results the resolution of templates should increase also. The number of objects with $z>1.5$ is small, so these requirements can be satisfied only if we reduce the number of eigenspectra from 3 to 2 . The comparison of the spectroscopic and photometric redshifts can be seen on Figure 8. There is one extreme outlier and there seems to be some systematic underestimation of redshift for high $z$ objects. Despite of these errors the redshift estimation is better than the one with the CWW templates. The rms error is $\sigma_{z}=0.34$ with our estimated and $\sigma_{z}=0.40$ with the CWW templates. The few outliers are responsible for most of the error. If we remove the one (three) extreme outlier(s) with $\Delta z>1$ we got $\sigma_{z}=0.17$ $\left(\sigma_{z}=0.22\right) \mathrm{rms}$ dispersion with the estimated (CWW) templates, respectively.

\section{Discussion and Applications}

The technique we described above incorporates the strengths of the empirical and template based photometric redshift techniques. It does this by utilizing a training set of galaxies (with colors and redshifts) to derive a set of spectral energy distributions (as opposed to defining a general but somewhat arbitrary set of polynomial coefficients). The result of this optimization procedure is to produce galaxy spectra that have been optimized to match the color distribution of galaxies within a given sample. It is important to note that the spectra are not optimized to produce the spectroscopic redshifts of the training set and, therefore, a comparison between the observed (spectroscopic) and predicted (photometric) redshifts is a statistically fair comparison. As we have defined a physical basis for the photometric-redshift relation (as opposed to the general polynomial relation) we can apply the spectral energy distributions over a wide range in redshift and are not restricted to just the redshift range over which we derive the relation (which was a fundamental limitation on our earlier approach).

As we have shown in Figure 7 applying this technique we reduce the scatter about the photometric-redshift relation to $\sigma_{z}=0.077$. This compares favorably with the results obtained 
by Fernandez-Soto et al (1999) who obtain a dispersion of $\sigma_{z}=0.095$ for galaxies with $z<1.35$. The reason for the decrease in the dispersion about the photometric-redshift relation is the improvement in the spectrophotometric templates used for estimating the galaxy redshifts. The standard Coleman, Wu and Weedman (1980) templates, while producing a remarkably good fit, are based on the spectra of approximately 12 local galaxies. The optimization technique we employ utilizes the colors of 74 galaxies (distributed over a range of redshifts) and is, therefore, more likely to sample the full distribution of galaxy types.

The limitation of our current application is simply the number of galaxies with accurate multicolor photometry in the Hubble Deep Field. The optimization technique is, however, general enough that we can incorporate any multicolor photometric and spectroscopic survey into the analysis. With the new generation of multicolor redshift surveys nearing completion we expect this approach to substantially improve on standard template-based photometric-redshift relations over the next few years. It should also be noted that as the result of this analysis is also a set of spectral energy distributions (or more exactly a statistical representation of the spectra) the eigenspectra can be applied to new multicolor surveys without requiring that they be transformed to the same photometric system.

As the spectral energy distributions we reconstruct are derived directly from observations they include the effects of dust and galaxy evolution. It is reasonable to expect that these spectra will be a better representation of galaxies over a wide range in redshift than standard local galaxy templates (as noted above). The derived eigentemplates can therefore be used to construct a set of K-corrections optimized for galaxies over a wide range in redshift. Ultimately, with large photometric samples, we can take this analysis one step further. Comparing the predictions of spectral synthesis models with those derived from the multicolor photometry will show how star formation and reddening by dust couple to produce the observed colors of galaxies. It should, therefore, be possible to identify where the models of star formation and the observed spectral properties of galaxies deviate and thereby improve on the spectral synthesis codes.

Finally we note that the analysis we have currently undertaken requires the use of multicolor photometry on galaxies of known spectroscopic redshift. On can extend this analysis to the case of data with only multicolor observations (i.e. no spectroscopic redshifts). We could optimize the estimated fluxes not only for the expansion coefficients and for the shape of the eigenspectra as we described above but also for the redshifts. This would naturally increase the sample of galaxies available for the optimization procedure (enabling much finer resolution for the resultant eigenspectra). It would also become a correspondingly larger computational problem. Another possibility would be to use photometric rather than spectroscopic redshifts. 


\section{Conclusions}

We have presented a new technique that can reconstruct the continuum spectra of galaxies directly from a set of multicolor photometric observations and spectroscopic redshifts. Using simulated multicolor data we show that we can recover the underlying spectral energy distribution even in the presence of substantial amounts of noise. Applying this approach to existing optical and near-infrared photometric data from the Hubble Deep Field we derive a set of spectral energy distributions that describe the observed galaxy colors. The main spectral features present in the spectral energy distributions of galaxies could be clearly seen within the reconstructed low resolution eigenspectra. The utility of this approach is demonstrated by using the empirical spectral energy distributions in a template-based photometric-redshift relation. The photometric redshift estimation based on the resultant template spectra gives redshift errors significantly better than standard template techniques. The current limitation on the accuracy of our technique is simply the number of high signal-to-noise multicolor photometric data currently available. Given the new photometric and spectroscopic surveys underway or nearing completion we anticipate a significant improvement in the resolution and accuracy of the derived spectral energy distributions.

We would like to thank Daniel Eisenstein, Jim Annis and David Hogg for useful discussions about our reconstruction technique. IC acknowledges partial support from the MTA-NSF grant no. 124 and the Hungarian National Scientific Research Foundation (OTKA) grant no. T030836, AJC acknowledges support from an LTSA grant (NAG57934). AS acknowledges support from NSF (AST9802980) and a NASA LTSA (NAG53503). 


\section{REFERENCES}

Benitez, N., 1999, astro-ph/9811189, ApJ in press.

Bruzual, A.G. \& Charlot, S., 1993, ApJ, 405, 538

Budavári, T., Szalay, A.S., Connolly, A.J., Csabai, I. \& Dickinson, M.E., 1999, in preparation

Coleman, G.D., Wu., C.-C. \& Weedman, D.W., 1980, ApJS, 43, 393

Connolly, A.J., Csabai, I., Szalay, A.S., Koo, D.C., Kron, R.G. \& Munn, J.A., 1995a, AJ 110, 2655

Connolly A.J., Szalay A.S., Bershady M.A., Kinney A.L. \& Calzetti D., 1995b, AJ, 110, 1071

Dickinson, M.E. et al, 1999, in preparation

Fernandez-Soto, A., Lanzetta, K.M. \& Yahil, A., 1999, in press

Gwyn, S.D.J. \& Hartwick, F.D.A., 1996, ApJ, 468, L77

Karhunen, H., 1947, Ann. Acad. Science Fenn, Ser. A.I. 37

Lilly, S.J., Tresse, L., Hammer, F., Crampton, D. \& Le Fevre, O., 1995, ApJ, 455, 108

Loève, M., 1948, Processus Stochastiques et Mouvement Brownien, Hermann, Paris, France

Madau, P., Ferguson, H.C., Dickinson, M.E., Giavalisco, M., Steidel, C.C. \& Fruchter,A., 1996, MNRAS, 283, 1388

Sawicki, M.J., Lin, H. \& Yee, H.K.C., 1997, AJ, 113, 1

Williams, R.E. et al., 1996, AJ, 112, 1335 

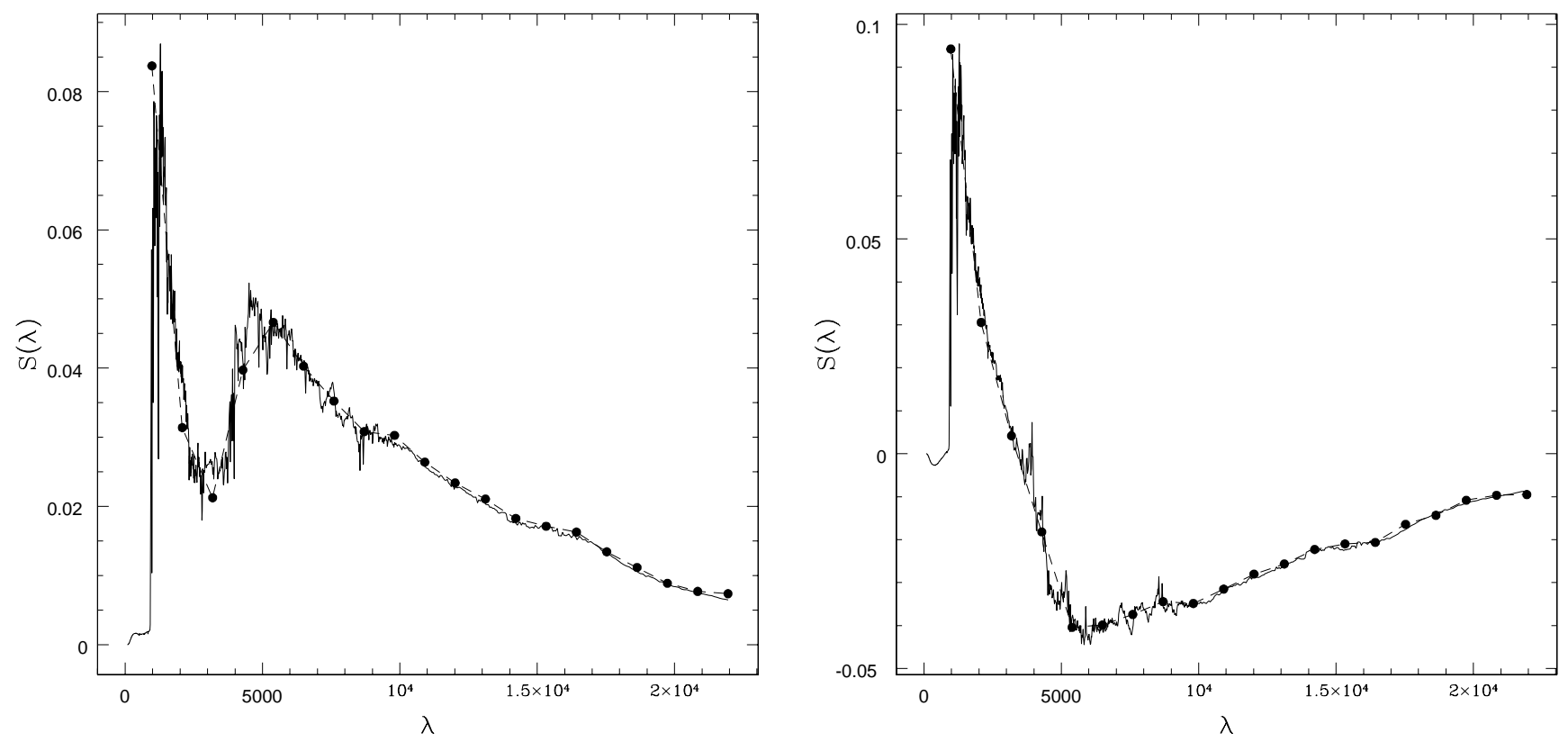

Fig. 1. - The left hand panel shows the first eigenspectrum (solid line) used to generate a simulated photometric catalog. The filled circles represent the reconstructed eigenspectrum derived from the multicolor photometry. The reconstruction was undertaken using 20 Legendre polynomials as the basis functions sampled at 20 points (i.e. with a resolution of approximately $1000 \AA$ ). The expansion coefficients, $b_{j l}$ (see Equation 3), for the Legendre polynomials were were initialized with random values. The reconstructed spectrum shown above was achieved after 50 iterations. The right hand panel shows the second eigenspectrum and the corresponding second reconstructed eigenspectrum. The rms deviation between the original and reconstructed eigenspectra is $6.98 \%$ and $6.99 \%$ for the first and second eigenspectra respectively 


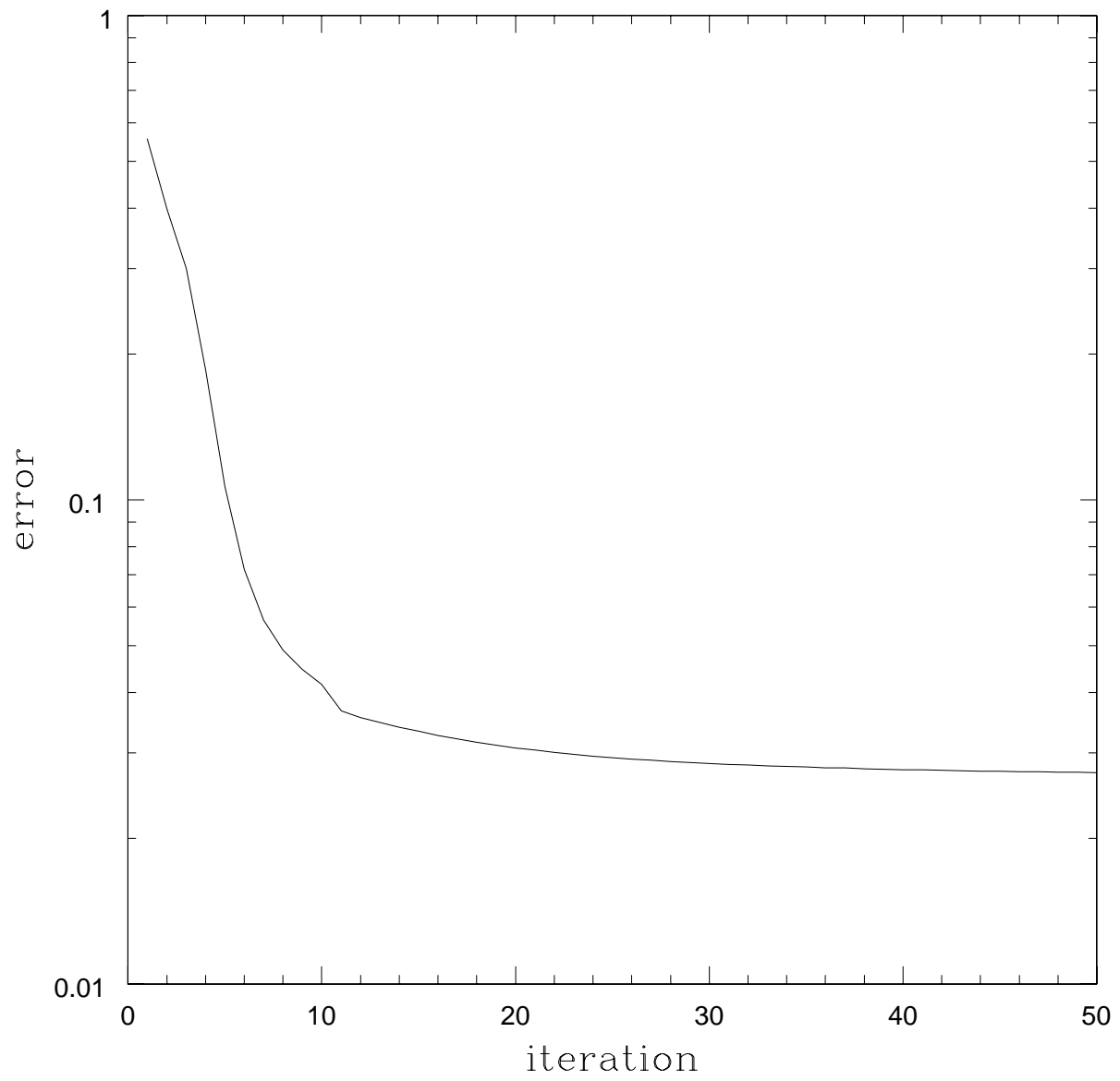

Fig. 2.- The convergence of the relative error as a function of the number of iterations for the reconstruction of the galaxy eigenspectra. The relative error decreases rapidly and by the 50th iteration varies by $<0.1 \%$ from one iteration to the next. 


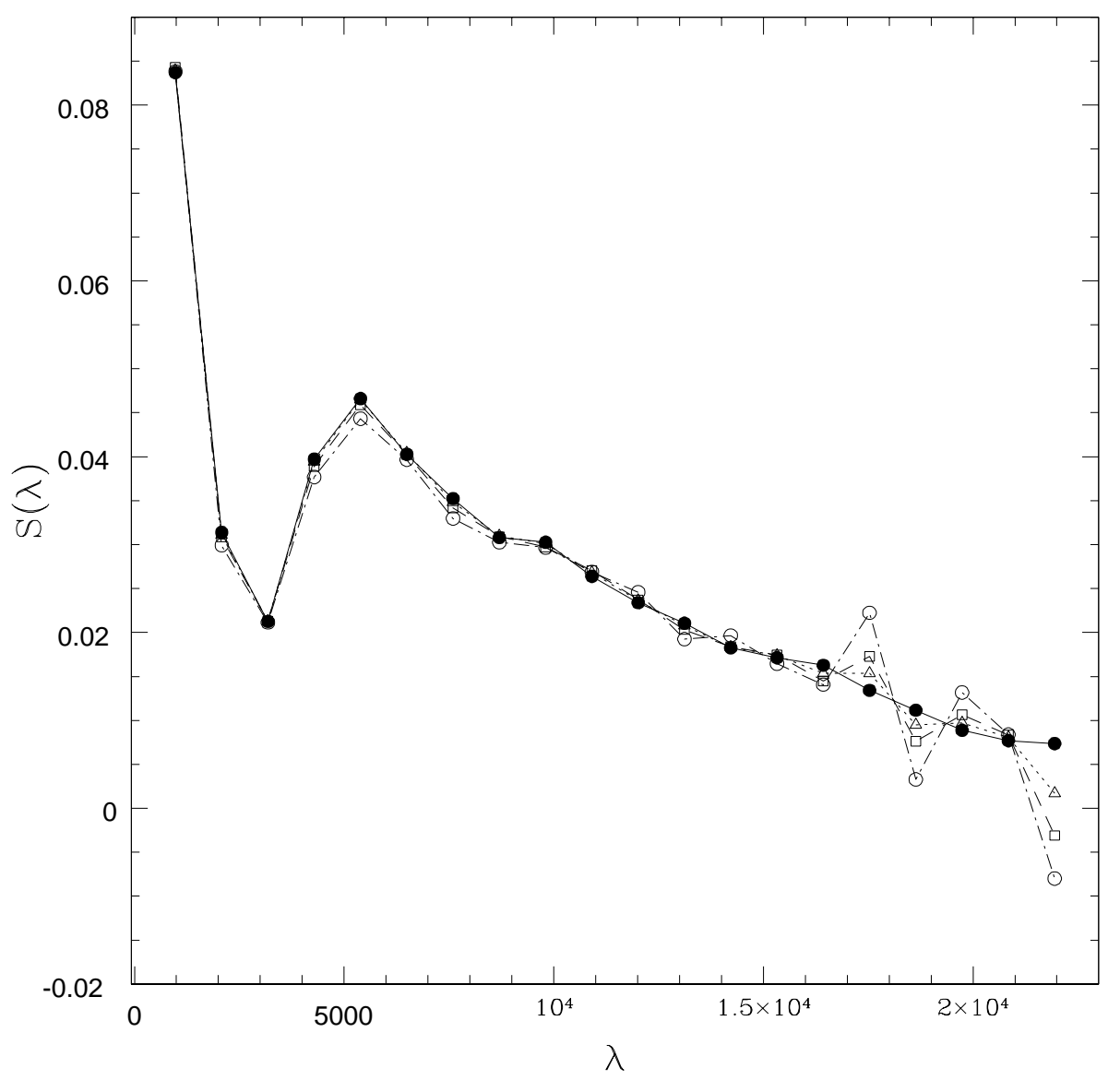

Fig. 3.- The effect of photometric uncertainty on the reconstruction of the first eigenspectrum. The filled circles show the reconstruction if there were no photometric errors within the data. The triangles, squares and open circles show the effect of increasing photometric errors within the data to $5 \%, 10 \%$ and $20 \%$ respectively. With a large number of galaxies the underlying eigenspectra that describe the galaxy distribution can be reconstructed to a high degree of accuracy even with relatively low signal-to-noise data. The multiplex advantage of having many galaxies sampling the same spectral intervals means that we have a resultant increase in the overall signal-to-noise of the output spectra (i.e. we are essentially coadding the data to beat down the noise). Since the long tails of the spectra are reconstructed using information only from highest and lowest redshift galaxies, the first signs of the error show up there. When calculating magnitudes the convolution with the filters partly averages out these fluctuations. 

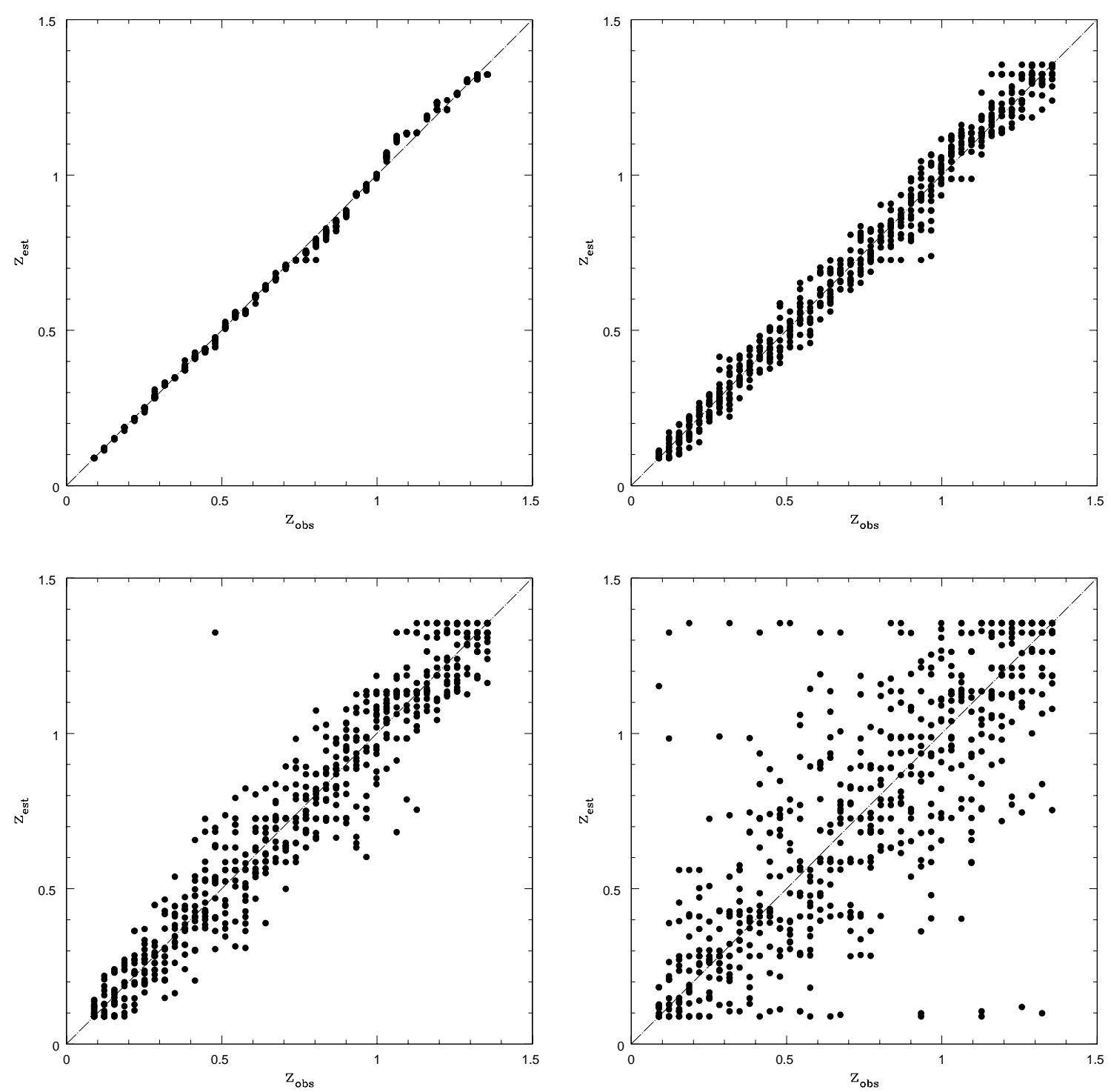

Fig. 4. - The photometric-redshift relation derived from the simulated data set using a range of signal-to-noise ratios. The panels, from left to right and top to bottom, show the effect of $0 \%, 5 \%$, $10 \%$ and $20 \%$ errors in the photometric data. For the analysis it was assumed that each of the photometric passbands had an identical signal-to-noise. 


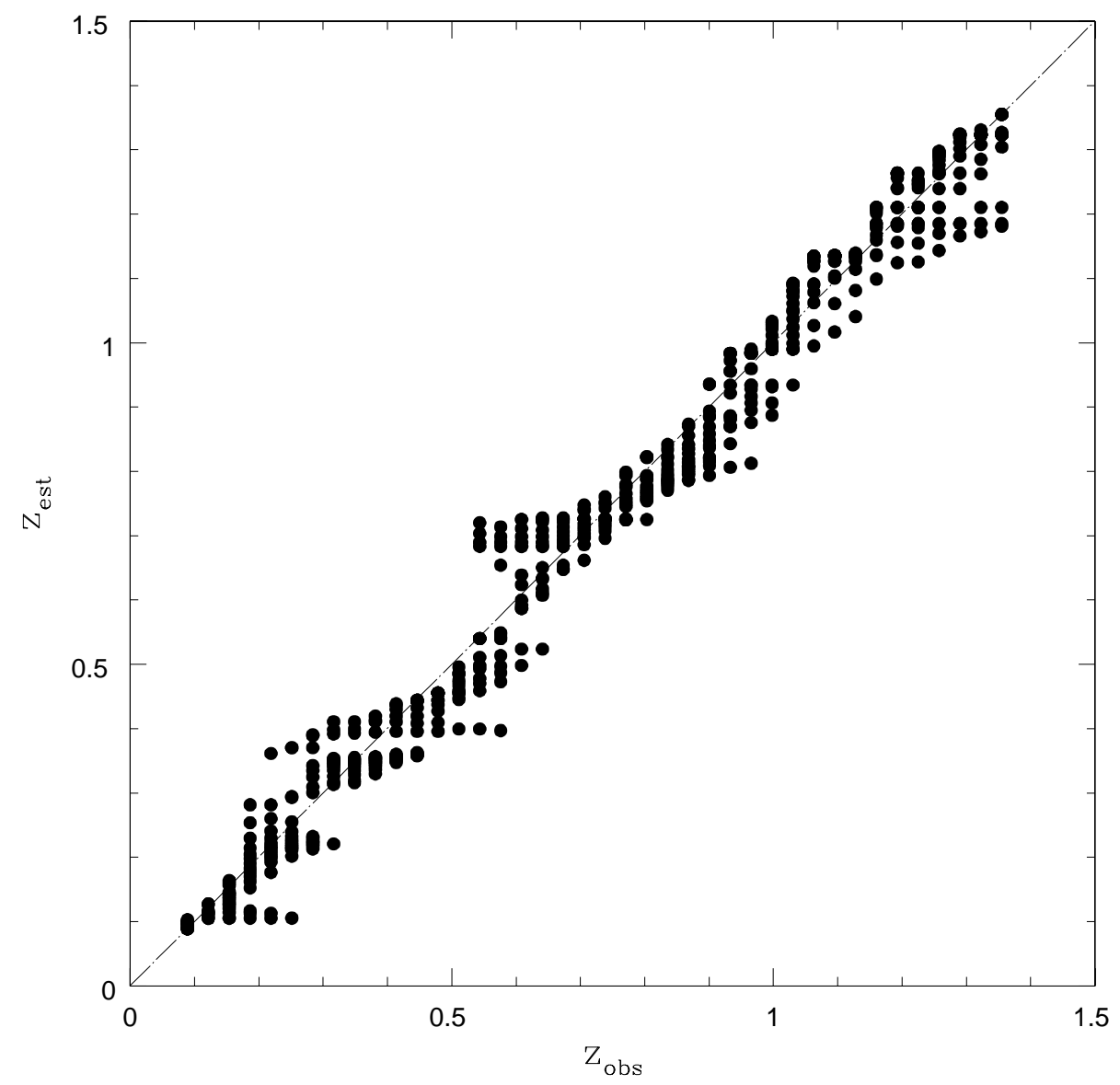

Fig. 5. - The photometric redshift relation for the simulated data set where the eigenspectra have been defined using a set of photometric data with $20 \%$ errors in the flux and then applied to a data set with $0 \%$ error in the flux. The dispersion about this relation is $\sigma_{z}=0.05$ which is comparable to the $\sigma_{z}=0.02$ found for the ideal dataset. This validates the analysis shown in Figure 3 where, even with large photometric errors, we can reconstruct the underlying eigensystem with a very high degree of accuracy. The uncertainty in the resultant photometric redshift relation is, therefore, dominated by the signal-to-noise of the data we wish to apply it to. 


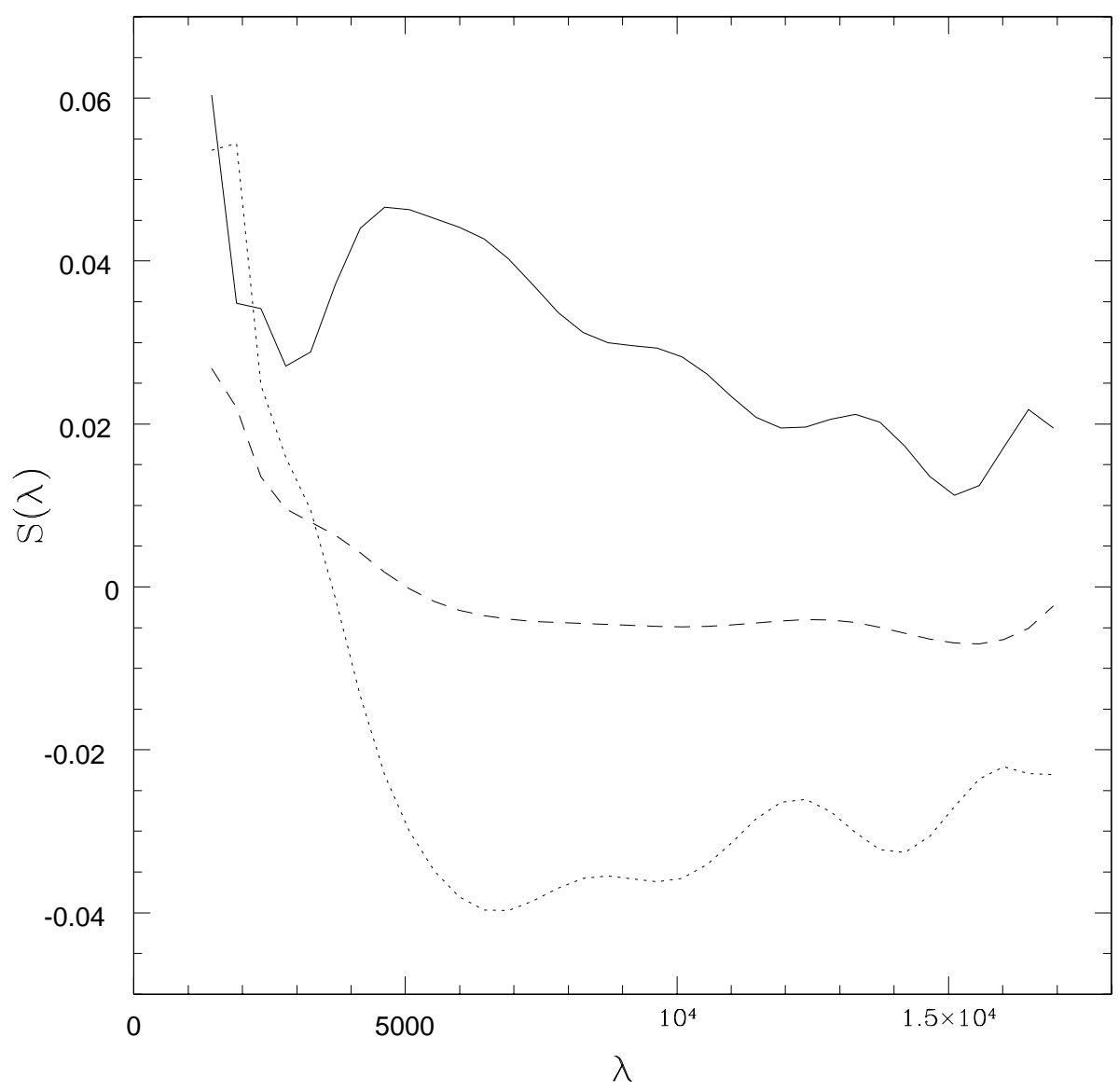

Fig. 6. - The HDF catalog with 74 galaxies $z<1.35$ were used to reconstruct spectral templates. The eigentemplates were iterated from random parameters using an orthogonal base of 20 Legendre polynomials (sampled at $450 \AA$ resolution). The solid line shows the first eigenspectrum derived from the data and the dashed and dotted lines the second and third eigenspectra respectively. Even given the low resolution nature of the output spectra the break at $4000 \AA$ and the rise in the ultraviolet continuum due to the presence of star formation are clearly visible. 


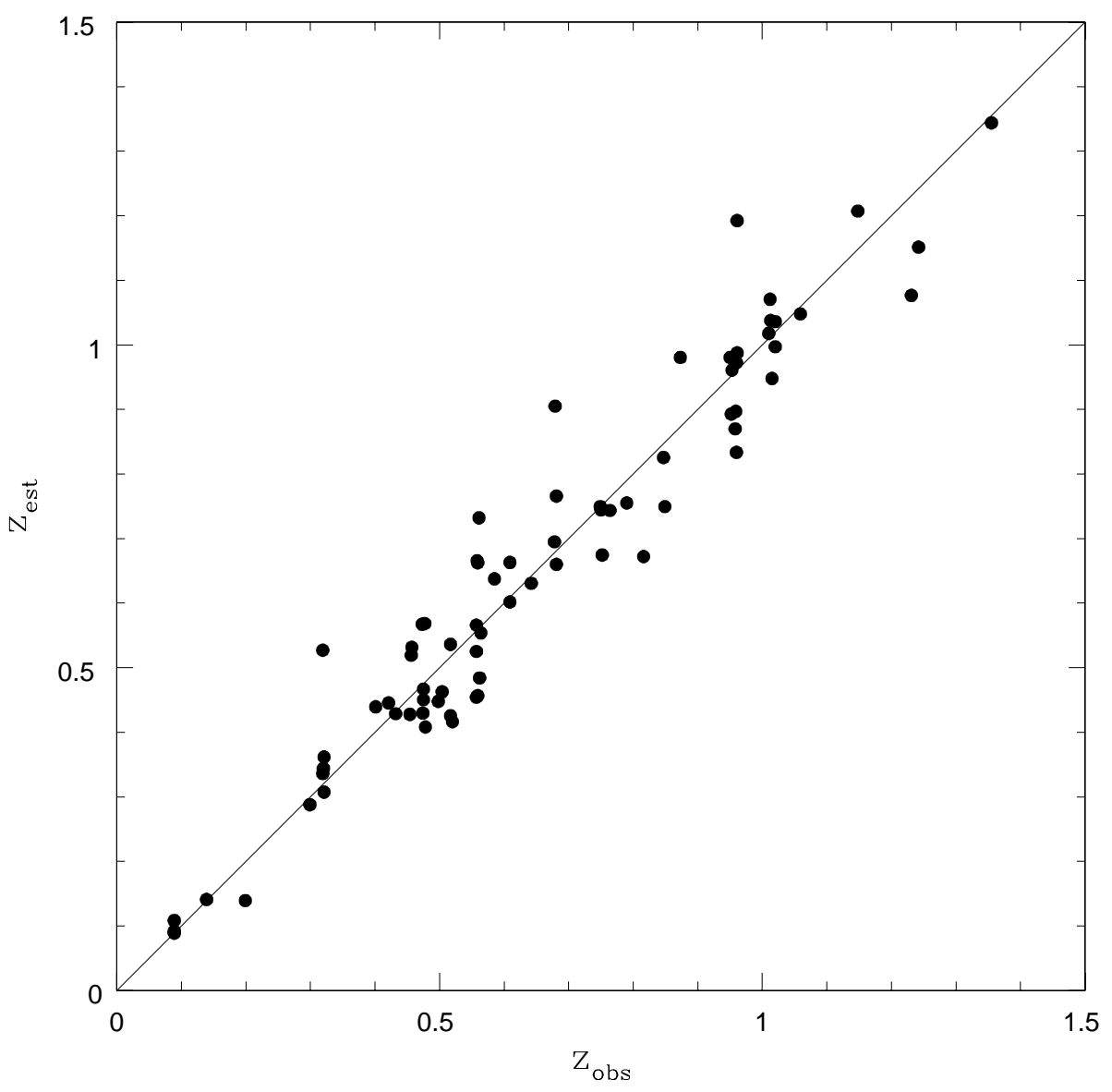

Fig. 7.- The photometric redshift estimation using the three reconstructed eigenspectra from the HDF photometric catalog (see Figure 4). This analysis gives a smaller dispersion in the photometric redshifts relation, $\sigma_{z}=0.077$, than that derived by Fernandez-Soto et. al. (1999), $\sigma_{z}=0.095$, using the four Coleman, Wu and Weedman (1980) (CWW) model/empirical spectral energy distributions. Polynomial photometric redshift estimation technique (Connolly et al 1995) gave $\sigma_{z}=0.14$ and $\sigma_{z}=0.062$ with first and second order polynomials respectively. 


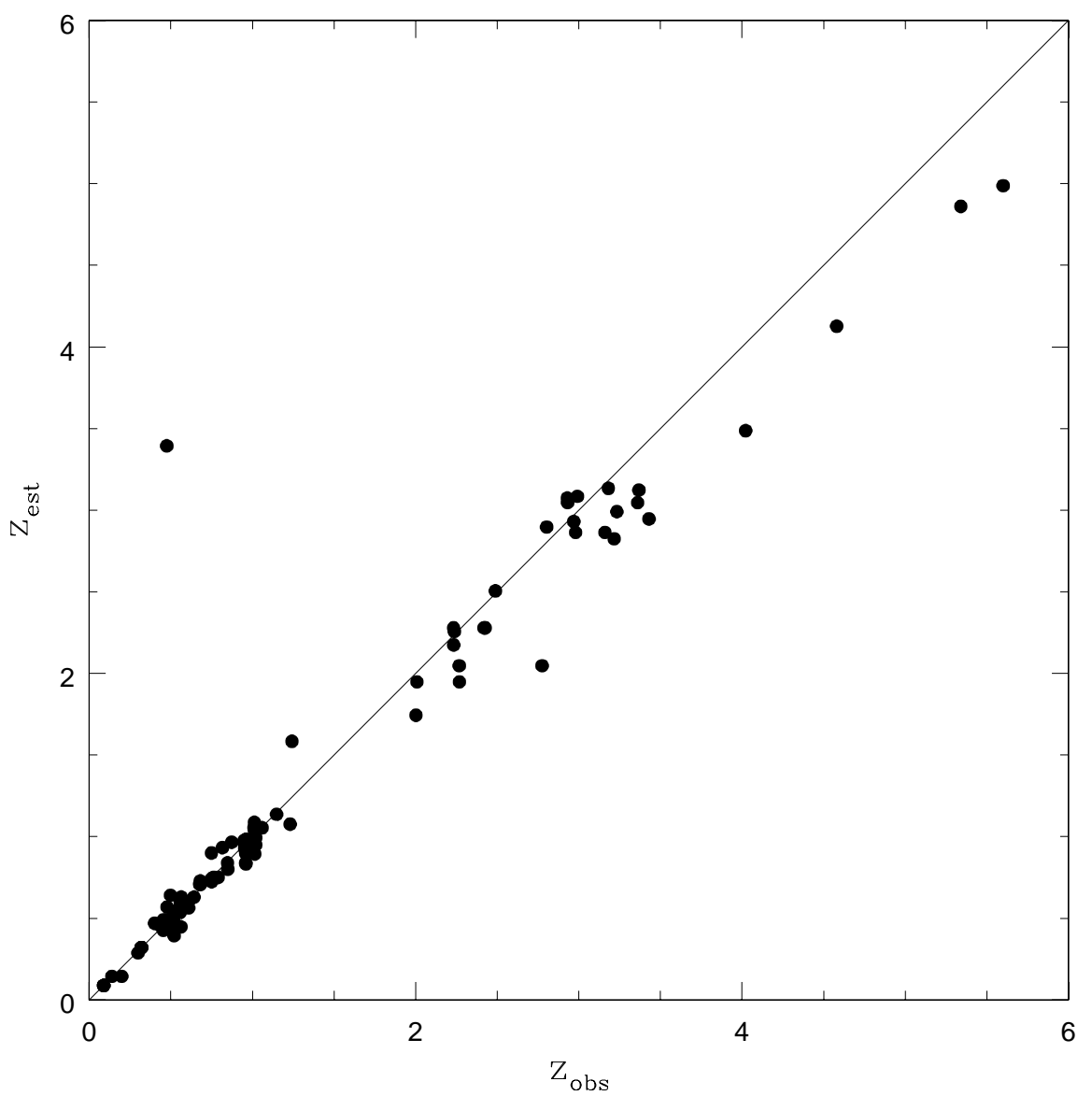

Fig. 8.- The photometric redshift estimation using two reconstructed eigenspectra from the HDF photometric catalog including high redshift objects also. Despite of the small number of high redshift objects in the training set the estimated templates give better photometric redshifts $\left(\sigma_{z}=0.34\right)$, than that derived by Fernandez-Soto et. al. (1999), $\left(\sigma_{z}=0.40\right)$, using the CWW spectral energy distributions. Removing the extreme outliers $(\Delta z>1.0)$ the dispersion decreases to $\sigma_{z}=0.17$ and $\sigma_{z}=0.22$ for the estimated and CWW templates respectively. 